\title{
LOCALIZATION LEMMAS FOR THE BERGMAN METRIC AT PLURISUBHARMONIC PEAK POINTS
}

\author{
GREGOR HERBORT
}

\begin{abstract}
Let $D$ be a bounded pseudoconvex domain in $\mathbb{C}^{n}$ and $\zeta \in \mathrm{D}$. By $K_{D}$ and $B_{D}$ we denote the Bergman kernel and metric of $D$, respectively. Given a ball $B=B(\zeta, R)$, we study the behavior of the ratio $K_{D} / K_{D \cap B}(w)$ when $w \in D \cap B$ tends towards $\zeta$. It is well-known, that it remains bounded from above and below by a positive constant. We show, that the ratio tends to 1 , as $w$ tends to $\zeta$, under an additional assumption on the pluricomplex Green function $\mathcal{G}_{D}(\cdot, w)$ of $D$ with pole at $w$, namely that the diameter of the sublevel sets $A_{w}:=\left\{z \in D \mid \mathcal{G}_{D}(z, w)<-1\right\}$ tends to zero, as $w \longrightarrow \zeta$. A similar result is obtained also for the Bergman metric. In this case we also show that the extremal function associated to the Bergman kernel has the concentration of mass property introduced in [DiOh1], where the question was discussed how to recognize a weight function from the associated Bergman space. The hypothesis concerning the set $A_{w}$ is satisfied for example, if the domain is regular in the sense of Diederich-Fornæss, ([DiFo2]).
\end{abstract}

\section{$\S 1$. Introduction}

Let $D$ be a bounded pseudoconvex domain in $\mathbb{C}^{n}$ with Bergman kernel $K_{D}$ and Bergman metric $B_{D}$. We fix a boundary point $\zeta$ of $D$. For a ball $B=B(\zeta, R)$ we study the ratios

$$
Q_{D, B}(w):=\frac{K_{D}}{K_{D \cap B}}(w)
$$

and

$$
\widehat{Q}_{D, B}(w ; X):=\frac{B_{D}}{B_{D \cap B}}(w ; X)
$$

at a point $w \in D \cap B$ and a unit vector $X \in \mathbb{C}^{n}$ and ask:

Under which reasonable assumption on $\zeta$ do these ratios tend to 1 as $w \longrightarrow \zeta$ ?

Received November 20, 2001.

Revised June 10, 2002.

2000 Mathematics Subject Classification: 32A36, 32F45, 32 U35. 
Also, since the work of Hörmander, [Hoer], we know that $Q_{D, B}(w) \longrightarrow$ 1 , for $w \longrightarrow \zeta$, if $\zeta$ is a local holomorphic peak point. For an alternative proof of this fact (using purely Stein theoretic methods), which yields also the corresponding result for the ratio $\widehat{Q}_{D, B}(w ; X)$, see [Di].

If $D$ is a general bounded pseudoconvex domain the ratio $Q_{D, B}(w)$ was in [Oh1] shown to be bounded from above and below by certain positive constants for an arbitrary boundary point $\zeta \in \partial D$. The corresponding result for $\widehat{Q}_{D, B}(w ; X)$ was obtained in [DiFoHe]. In Proposition 3.2 of [McN] one can find a localization result (in the small-constant-large-constant sense) for the more general domain functionals $\mathcal{M}_{D}(\cdot ; M, Y ; w)$ that will be introduced below.

In $[\mathrm{Nik}]$ it is implicitly shown, that on an arbitrary (i.e. not necessarily bounded) pseudoconvex domain the ratios $Q_{D, B}(w)$ and $\widehat{Q}_{D, B}(w ; X)$ remain bounded from above and below by positive constants near any plurisubharmonic peak point $\zeta \in \partial D$.

If one wants to prove $\lim _{w \rightarrow \zeta} Q_{D, B}(w)=1$, or $\lim _{w \rightarrow \zeta} \widehat{Q}_{D, B}(w ; X)=1$, the assumption that $\zeta$ is a local holomorphic peak point is felt to be very restrictive. It is known to be satisfied in classical cases (strongly pseudoconvex case, points of finite type in $\mathbb{C}^{2}$ (see $[\mathrm{BeFo}]$ ) or of finite semiregular type (see [DiHe1], or [Yu])).

The most natural way to weaken it, is to require that $\zeta$ is a plurisubharmonic peak point. There is a wide class of pseudoconvex domains that includes the finite type domains. These domains are the so-called regular domains and were introduced in [DiFo2]. Intuitively speaking, a smooth bounded pseudoconvex domain $D$ is regular, if the Levi degeneracy set $E$ in $\partial D$ is stratified into certain subsets, each of which is locally contained in a smooth submanifold of $\partial D$ that has no complex tangents that are at the same time weakly pseudoconvex tangential directions. (In particular, no complex analytic varieties of positive dimension can be contained in $\partial D$ ). In [DiFo2] it was shown, that the closure of a regular pseudoconvex domain admits a Stein neighborhood basis. Later Sibony, [Si] proved that each boundary point $q$ of a regular domain $D$ is a peak point with respect to the family of continuous functions on $\bar{D}$, that are plurisubharmonic inside $D$.

Recently the pluricomplex Green function $\mathcal{G}_{D}(\cdot, w), w \in D$, has been successfully applied to questions in Bergman geometry, see [BlPf], [He1], [DiHe2].

This function is defined by

$$
\mathcal{G}_{D}(\cdot, w):=\sup \{u(z) \mid u \in P(w ; D)\}
$$

where $P(w ; D)$ denotes the class of all negative plurisubharmonic functions on $D$ such that $u-\log |\cdot-w|$ is bounded from above near $w$. 
It has been introduced and studied by Klimek, [Kli], and Demailly, [De] (for more general hyperconvex domains), who proved fundamental properties (in particular its relationship to the Monge-Ampère operator).

Its importance is based upon the fact that it has a logarithmic pole at $w$ and hence becomes an important tool in applying the $L^{2}$-theory for the $\bar{\partial}$-operator with plurisubharmonic weight functions, see [Hoer], [OhTa]. The $\bar{\partial}$-method is the main ingredience in the construction of holomorphic $L^{2}$-functions that exhibit a prescribed behavior at the point $w$.

What is needed, however, is information about the boundary behavior of $\mathcal{G}_{D}(\cdot, w)$ under approach of $w$ to the boundary. This has been studied in [CCW], [He2].

A reasonable condition for (in a sense ) optimal localization lemmas for the quantities from Bergman theory can be stated in terms of the sublevel sets of the pluricomplex Green function $\mathcal{G}_{D}(\cdot, w)$ of $D$ with pole at $w$, see condition (1.1) below.

In Bergman theory domain functionals play a key role. By $H^{2}(D)$ we denote the Hilbert space of all holomorphic functions over $D$ that are square-integrable with respect to the Lebesgue measure.

Let for a multi-index $\alpha \in I N^{n}$ the symbol $D^{\alpha}$ denote the partial derivative $D^{\alpha}=\frac{\partial^{\alpha}}{\partial z^{\alpha}}$.

Given a $k$-tuple $M=\left(T_{1}, \ldots, T_{k}\right)$ of differential operators with constant coefficients and a $k$-tuple $Y:=\left(Y_{1}, \ldots, Y_{k}\right)$ of complex numbers, we denote for fixed $w \in D$ by $\mathcal{M}_{D}(\cdot ; M, Y ; w)$ the well-defined function from $H^{2}(D)$ having minimal $L^{2}$-norm among those functions $f \in H^{2}(D)$ satisfying the auxiliary conditions $T_{l} f(w)=Y_{l}$ for all $l=1,2, \ldots, k$. (We will suppose that the set of such functions is not empty).

Here are two well-known examples:

a) The function $K_{D}(\cdot, w) / K_{D}(w, w)$ can be represented as $\mathcal{M}_{D}(\cdot ; M$, $\{1\} ; w)$, where $M=\{T\}$, with $T f:=f$. Furthermore,

$$
\left\|\mathcal{M}_{D}(\cdot ; Y, M ; w)\right\|^{2}=1 / K_{D}(w, w) .
$$

b) Let $X$ denote a unit vector in $\mathbb{C}^{n}$. Now we choose $M=\left\{T_{1}, T_{2}\right\}$, where $T_{1} f=f$ and $T_{2} f=\sum_{j=1}^{n} X_{j} \frac{\partial f}{\partial z_{j}}$. Let $Y_{1}=0, Y_{2}=1$. Then

$$
\left\|\mathcal{M}_{D}(\cdot ; M, Y ; w)\right\|^{2}=\frac{1}{K_{D}(w, w) B_{D}^{2}(w ; X)} .
$$

Also, the holomorphic curvatures for the Bergman metric can be expressed in terms of such domain functionals. (See $[\mathrm{KrYu}]$ for domain functionals that decribe the Ricci or scalar curvature of the Bergman metric).

For $w \in D$ we denote by $A_{w}$ the sublevel set

$$
A_{w}:=\left\{z \in D \mid \mathcal{G}_{D}(z, w)<-1\right\} .
$$


Now our comparison theorem for the domain functionals is as follows:

Theorem 1.1. Let $D \subset \subset \mathbb{C}^{n}$ be pseudoconvex, and $\zeta \in \partial D$ a point such that

$$
\lim _{w \rightarrow \zeta} \operatorname{diam}\left(A_{w}\right)=0 .
$$

If $M$ and $Y$ have the meanings as explained before, then for any radius $R>0$ we have

$$
\lim _{w \rightarrow \zeta} \frac{\left\|\mathcal{M}_{D}(\cdot ; M, Y ; w)\right\|}{\left\|\mathcal{M}_{D \cap B(\zeta, R)}(\cdot ; M, Y ; w)\right\|}=1
$$

The method applied in the proof also gives the following result concerning the concentration of the $L^{2}$-mass of the minimizing function from Theorem 1.1 .

TheOREm 1.2. Let $D \subset \subset \mathbb{C}^{n}$ be pseudoconvex, and $\zeta \in \partial D$ a point such that

$$
\lim _{w \rightarrow \zeta} \operatorname{diam}\left(A_{w}\right)=0
$$

If $M$ and $Y$ have the meanings as explained before, then, given a radius $R>0$ and a number $s \in(0,1)$, there exists a number $\delta(R, s)>0$, such that

$$
\frac{\left\|\mathcal{M}_{D}(\cdot ; M, Y ; w)\right\|_{D \cap B(\zeta, R)^{C}}}{\left\|\mathcal{M}_{D}(\cdot ; M, Y ; w)\right\|}<s
$$

whenever $|w-\zeta|<\delta(R, s)$.

Here, by $S^{C}$ we always mean the complement of a set $S$.

Remarks. The above theorems do not explicitly require smoothness of the boundary of $D$ near $\zeta$.

The crucial property described in (1.1) is satisfied for example on regular domains, see [DiHe2].

The appearance of analytic discs in the boundary is certainly an obstacle for (1.1) in general, as the example of a bidisc shows. 


\section{§2. The proofs}

We have to show the following Main Lemma:

Main Lemma. Under the assumptions of Theorems 1.1 and 1.2 the following holds:

Given two numbers $s, R>0$ one can find a radius $\delta=\delta(s, R)>0$ with the following property:

For any point $w \in D \cap B(\zeta, \delta)$ and any function $f \in H^{2}(D \cap B(\zeta, R))$ there exists a function $\widetilde{f}_{w} \in H^{2}(D)$ such that

$$
T_{j} \widetilde{f}_{w}(w)=T_{j} f(w), \forall 1 \leq j \leq k
$$

and

$$
\left\|\tilde{f}_{w}\right\| \leq(1+s)\|f\|_{D \cap B(\zeta, R)} .
$$

It is (2.1) what requires $w$ to be situated close to $\zeta$.

We first show how Theorems 1.1 and 1.2 follow from the Main Lemma.

To obtain Theorem 1.1 we choose $f=\mathcal{M}_{D \cap B(\zeta, R)}(\cdot ; M, Y, w)$. If then $w \in D \cap B(\zeta, \delta)$, we note that $\tilde{f}_{w}$ yields a candidate for $\left\|\mathcal{M}_{D}(\cdot ; M, Y, w)\right\|$, and

$$
\begin{aligned}
\left\|\mathcal{M}_{D}(\cdot ; M, Y, w)\right\| & \leq\left\|\widetilde{f}_{w}\right\| \\
& \leq(1+s)\|f\|_{D \cap B(\zeta, R)} \\
& =(1+s)\left\|\mathcal{M}_{D \cap B(\zeta, R)}(\cdot ; M, Y, w)\right\| .
\end{aligned}
$$

Hence

$$
1 \leq \frac{\left\|\mathcal{M}_{D}(\cdot ; M, Y ; w)\right\|}{\left\|\mathcal{M}_{D \cap B(\zeta, R)}(\cdot ; M, Y ; w)\right\|} \leq 1+s
$$

for $w \in D \cap B(\zeta, \delta)$.

In a similar way we obtain Theorem 1.2, namely:

We apply the Main Lemma to the function $f=\mathcal{M}_{D}(\cdot ; M, Y, w)$ and get for points $w \in D \cap B(\zeta, \delta)$ by means of the function $\widetilde{f}_{w}$ :

$$
\begin{aligned}
\| \mathcal{M}_{D}(\cdot ; M & Y ; w) \|_{D \cap B(\zeta, R)^{C}} \\
= & \left\|\mathcal{M}_{D}(\cdot ; M, Y ; w)\right\|-\left\|\mathcal{M}_{D}(\cdot ; M, Y ; w)\right\|_{D \cap B(\zeta, R)} \\
\leq & \left\|\widetilde{f}_{w}\right\|-\left\|\mathcal{M}_{D}(\cdot ; M, Y ; w)\right\|_{D \cap B(\zeta, R)} \\
\leq & (1+s)\left\|\mathcal{M}_{D}(\cdot ; M, Y ; w)\right\|_{D \cap B(\zeta ; R)} \\
& \quad-\left\|\mathcal{M}_{D}(\cdot ; M, Y ; w)\right\|_{D \cap B(\zeta, R)} \\
= & s\left\|\mathcal{M}_{D}(\cdot ; M, Y ; w)\right\|_{D \cap B(\zeta, R)}
\end{aligned}
$$


This implies

$$
\frac{\left\|\mathcal{M}_{D}(\cdot ; M, Y ; w)\right\|_{D \cap B(\zeta, R)^{C}}}{\left\|\mathcal{M}_{D}(\cdot ; M, Y ; w)\right\|_{D}} \leq \frac{\left\|\mathcal{M}_{D}(\cdot ; M, Y ; w)\right\|_{D \cap B(\zeta, R)^{C}}}{\left\|\mathcal{M}_{D}(\cdot ; M, Y ; w)\right\|_{D \cap B(\zeta, R)}} \leq s
$$

as desired.

\section{Proof of the Main Lemma}

Our aim is to construct the desired function $\tilde{f}_{w} \in H^{2}(D)$ in the form

$$
\widetilde{f}_{w}(z):=\lambda_{\varepsilon}\left(\frac{|z-\zeta|}{2 R}\right) f(z)-u_{w, \varepsilon}(z)
$$

where $\lambda_{\varepsilon}$ is a suitable cut-off function (that will be constructed in the next subsection) and $u_{w, \varepsilon}$ is smooth and vanishes to sufficiently high order at $w$, while, at the same time its $L^{2}$-norm tends to zero, as $\varepsilon \searrow 0$. This function $u_{w, \varepsilon}$ will be gained by means of the $L^{2}$-techniques for solving the $\bar{\partial}$-equation with plurisubharmonic weights, as developped in [OhTa]. In that paper an apriori formula for the $\bar{\partial}$ operator at the level of $(n, 1)$-forms was derived on complex manifolds with a complete Kähler metric. This in conjunction with a standard Hahn-Banch argument made it possible to solve the $\bar{\partial}$-equation in the Kähler manifold setting. We will use a variant of this approach that works directly for $(0,1)$-forms on a pseudoconvex domain and was derived in $[\mathrm{DiHe} 3]$.

\section{A) A suitable cut-off function}

We make use of a cutoff-function, that can be obtained by modifying Lemma 2.2 from [DiOh2].

LEMMA 2.1. There exists a number $\varepsilon_{0}>0$ and a positive $M$, such that: For any $0<\varepsilon<\varepsilon_{0}$ there exists a smooth function $\lambda_{\varepsilon}: I R \longrightarrow[0,1]$, such that $\lambda_{\varepsilon}(t)=1$ for $t \leq \varepsilon e^{-2 \varepsilon}$ and $\lambda_{\varepsilon}(t)=0$ for $t \geq 1 / 3$, and furthermore the derivative is estimated by

$$
\left|\lambda_{\varepsilon}^{\prime}(t)\right| \leq \frac{M}{\log \log (1 / \varepsilon)} \frac{1}{t|\log t|}
$$

For the reader's convenience we give a proof of this. 
Proof. For $\varepsilon>0$ and $x<0$ we define a continuous function $h_{\varepsilon}$ by

$$
\begin{aligned}
& h_{\varepsilon}(x):= \\
& \left\{\begin{aligned}
1, & \text { if } x<-\varepsilon+\log \varepsilon \\
1+\log \frac{\log (-x-2 \varepsilon)}{\log (-\varepsilon+\log (1 / \varepsilon))}, & \text { if }-\varepsilon+\log \varepsilon \leq x \leq-2 \varepsilon-(-\varepsilon+\log (1 / \varepsilon))^{1 / e} . \\
0, & \text { if } x>-2 \varepsilon-(-\varepsilon+\log (1 / \varepsilon))^{1 / e}
\end{aligned}\right.
\end{aligned}
$$

On the open intervall $I_{\varepsilon}:=\left(-\varepsilon+\log \varepsilon,-2 \varepsilon-(-\varepsilon+\log (1 / \varepsilon))^{1 / e}\right)$ this function is smooth, and

$$
h_{\varepsilon}^{\prime}(x)=\frac{1}{(x+2 \varepsilon) \log (-x-2 \varepsilon)}, \quad h_{\varepsilon}^{\prime \prime}<0 .
$$

In particular, we have $-x \geq 2 \varepsilon+(-\varepsilon+\log (1 / \varepsilon))^{1 / e}$, for all $x \in I_{\varepsilon}$, and hence

$$
\left|h_{\varepsilon}^{\prime}(x)\right|=\frac{1}{|x+2 \varepsilon| \log (-x-2 \varepsilon)} \leq \frac{2}{\log \left((-\varepsilon+\log (1 / \varepsilon))^{1 / e}\right)} \frac{1}{|x|},
$$

if $\varepsilon<e^{-2}$.

We now smooth out the function $h_{\varepsilon}$ : Let $\alpha \in \mathscr{C}_{0}^{\infty}(-1,1)$ be a nonnegative function with $\int_{-1}^{1} \alpha(s) d s=1$. Then we consider

$$
\mu_{\varepsilon}(x):=\frac{1}{\varepsilon} \int_{-\varepsilon}^{\varepsilon} h_{\varepsilon}(x-s) \alpha(s / \varepsilon) d s .
$$

It can be left to the reader to show, that the function $\mu_{\varepsilon}$ satisfies:

$$
\begin{aligned}
& \mu_{\varepsilon}(x)=1, \text { on }(-\infty,-2 \varepsilon+\log \varepsilon), \\
& \mu_{\varepsilon}(x)=0, \text { on }\left(-\varepsilon-(-\varepsilon+\log (1 / \varepsilon))^{1 / e}, \infty\right)
\end{aligned}
$$

and that, furthermore, with some constant $M>0$, one has

$$
\left|\mu_{\varepsilon}^{\prime}(x)\right| \leq \frac{M}{\log \log (1 / \varepsilon)} \frac{1}{|x|}
$$

everywhere. The requirements of the lemma will now be fulfilled by

$$
\lambda_{\varepsilon}(t)=\mu_{\varepsilon}(\log t), \quad t>0
$$




\section{B) Solving the $\bar{\partial}$-equation with weights}

Let $U \subset \mathbb{C}^{n}$ be an open set and $\varphi \in \mathscr{C}^{0}(U)$. We denote by $L^{2}(U, \varphi)$ the space of all measurable functions $f$ on $U$, for which the weighted $L^{2}$ integral

$$
\|f\|_{\varphi}:=\left(\int_{U}|f|^{2} e^{-\varphi} d^{2 n} z\right)^{1 / 2}
$$

is finite (here, $d^{2 n} z$ is the Lebesgue measure in $\mathbb{C}^{n}$ ). Let $L_{q}^{2}(U, \varphi)$ be the space of $(0, q)$ forms with coefficients in $L^{2}(U, \varphi)$. These spaces are Hilbert spaces, the inner product being defined by

$$
(u, v)_{\varphi}:=\int_{U} u \wedge \bar{\star} v e^{-\varphi}
$$

where $\star$ is the euclidean Hodge star operator.

We will use some more notation:

For a $\mathscr{C}^{2}$-function $h$ on $U$ we write $\mathscr{L}_{h}$ for the Levi form of $h$.

If $Q=\sum_{a, b=1}^{n} Q_{a \bar{b}} d z_{a} d \bar{z}_{b}$ is a hermitian form defined over $U$ we write $Q(u):=\sum_{a, b=1}^{n} Q_{a \bar{b}} u_{a} \overline{u_{b}}$ for any $(0,1)$-form $u=\sum_{k=1}^{n} u_{k} d \bar{z}_{k}$ on $U$, and, if $Q$ is positive definite, we denote by $Q^{-1}(u)$ the form $Q^{-1}(u):=\sum_{a, b=1}^{n} Q^{a \bar{b}} u_{a} \overline{u_{b}}$, where $Q^{a \bar{b}}$ describe the entries of $\left(Q_{a \bar{b}}\right)_{a, b=1}^{n}$.

With these notations we state our solution lemma for the $\bar{\partial}$-equation as follows

Lemma 2.2. Let $\Omega \subset \subset \mathbb{C}^{n}$ be a pseudoconvex domain with a $\mathscr{C}^{2}$ boundary. Suppose that on $\bar{\Omega}$ we are given two functions $\varphi$ and $\eta$ of class $\mathscr{C}^{\infty}$, where $\eta>0$ on $\bar{\Omega}$, that satisfy

$$
\eta \mathscr{L}_{\varphi}-\mathscr{L}_{\eta} \geq Q+\frac{1}{2} \frac{\partial \eta \otimes \overline{\partial \eta}}{\eta}
$$

where $Q$ is some positive definite hermitian form on $\Omega$.

Then, given a smooth $\bar{\partial}$-closed $(0,1)$-form $v$ on $\Omega$ satisfying

$$
J_{Q, \varphi}(v):=\int_{\Omega} Q^{-1}(v) e^{-\varphi} d^{2 n} z
$$

there exists a smooth solution $u$ to the equation

$$
\bar{\partial}(\sqrt{\eta} u)=v
$$

over $\Omega$, such that the growth condition

$$
\|u\|_{\varphi}^{2} \leq 10 J_{Q, \varphi}(v)
$$

is fulfilled. 
Proof. The operator $\bar{\partial}$ and its formal adjoint $\vartheta$ admit densely defined closures $\bar{\partial}$ and $\bar{\partial}_{\varphi}^{*}$ on the spaces $L_{q}^{2}(\Omega, \varphi), q=0,1$, respectively.

We want to show

$$
\left|(u, v)_{\varphi}\right|^{2} \leq 10 J_{Q, \varphi}(v)\left(\|\sqrt{\eta} \bar{\partial} u\|_{\varphi}^{2}+\left\|\sqrt{\eta} \bar{\partial}_{\varphi}^{*} u\right\|_{\varphi}^{2}\right)
$$

for all $u \in L_{1}^{2}(\Omega, \varphi) \cap \operatorname{dom}(\bar{\partial}) \cap \operatorname{dom}\left(\bar{\partial}_{\varphi}^{*}\right)$.

In order to achieve this we may restrict to forms in the space $\mathscr{F}$ of all $(0,1)$-forms $u=u_{1} d \bar{z}_{1}+\cdots+u_{n} d \bar{z}_{n}$ that have coefficients in $C^{1}(\bar{\Omega})$ and satisfy the Neumann condition $u \downarrow \partial r=0$ at the boundary of $\Omega$. (Here $r$ denotes a defining function for $\Omega$ ). This space is known to be densely situated in $L_{1}^{2}(\Omega, \varphi) \cap \operatorname{dom}(\bar{\partial}) \cap \operatorname{dom}\left(\bar{\partial}_{\varphi}^{*}\right)$ in the graph norm associated to $\bar{\partial}$ and $\bar{\partial}_{\varphi}^{*}$ (,see [Hoer], p. 100). Now, Lemma 2.1b) of [DiHe3] applies and gives for any form $u \in \mathscr{F}$ :

$$
\left.\|\sqrt{\eta} \bar{\partial} u\|_{\varphi}^{2}+\left\|\sqrt{\eta} \bar{\partial}_{\varphi}^{*} u\right\|_{\varphi}^{2} \geq \int_{\Omega}\left(\eta \mathscr{L}_{\varphi}-\mathscr{L}_{\eta}\right)(u) e^{-\varphi} d^{2 n} z+2 \operatorname{Re}(u\rfloor \partial \eta, \bar{\partial}_{\varphi}^{*} u\right)_{\varphi}
$$

Combining this with

$$
\begin{aligned}
2\left|\operatorname{Re}\left(u \_\partial \eta, \bar{\partial}_{\varphi}^{*} u\right)_{\varphi}\right| & \leq \| u\rfloor \partial \eta / \sqrt{\eta}\left\|_{\varphi}\right\| \sqrt{\eta} \bar{\partial}_{\varphi}^{*} u \|_{\varphi} \\
& \left.\leq \frac{1}{4} \| u\right\rfloor \partial \eta / \sqrt{\eta}\left\|_{\varphi}^{2}+4\right\| \sqrt{\eta} \bar{\partial}_{\varphi}^{*} u \|_{\varphi}^{2} \\
& \leq \frac{1}{2} \int_{D_{t}}\left(\eta \mathscr{L}_{\varphi}-\mathscr{L}_{\eta}\right)(u) e^{-\varphi} d^{2 n} z+4\left\|\sqrt{\eta} \bar{\partial}_{\varphi}^{*} u\right\|_{\varphi}^{2}
\end{aligned}
$$

(the last estimate follows from (2.2))

we obtain together with our hypothesis (2.2) and the Cauchy-Schwarzinequality

$$
\begin{aligned}
\left|(u, v)_{\varphi}\right|^{2} & \leq J_{Q, \varphi}(v) \int_{\Omega} Q(u) e^{-\varphi} d^{2 n} z \\
& \leq J_{Q, \varphi}(v) \int_{\Omega}\left(\eta \mathscr{L}_{\varphi}-\mathscr{L}_{\eta}\right)(u) e^{-\varphi} d^{2 n} z \\
& \leq 10 J_{Q, \varphi}(v)\left(\|\sqrt{\eta} \bar{\partial} u\|_{\varphi}^{2}+\left\|\sqrt{\eta} \bar{\partial}_{\varphi}^{*} u\right\|_{\varphi}^{2}\right)
\end{aligned}
$$

for $u \in \mathscr{F}$; this gives us (2.3). The claim now follows from the Hahn-Banach theorem applied to the linear form $\mathscr{L}\left(\sqrt{\eta} \bar{\partial}_{\varphi}^{*} u\right):=(u, v)_{\varphi}$, which is now welldefined on the subspace $E:=\left\{\sqrt{\eta} \bar{\partial}_{\varphi}^{*} u \mid u \in L_{1}^{2}(\Omega, \varphi) \cap \operatorname{dom}(\bar{\partial}) \cap \operatorname{dom}\left(\bar{\partial}_{\varphi}^{*}\right)\right\}$. 


\section{C) Construction of the function $\widetilde{f}_{w}$}

Let $\left(D_{t}\right)_{t>0}$ be an exhaustion of $D$ by smooth bounded pseudoconvex subdomains. For each $t$ we choose on $D_{t}$ a regularization $\mathcal{G}_{D}^{(t)}(\cdot, w)$ of $\mathcal{G}_{D}(\cdot, w)$, (where $w \in D$ is arbitrary), such that $0 \geq \mathcal{G}_{D}^{(t)}(\cdot, w) \geq \mathcal{G}_{D}(\cdot, w)$ and $\mathcal{G}_{D}^{(t)}(\cdot, w) \searrow \mathcal{G}_{D}(\cdot, w)$, as $t \searrow 0$.

Let $L$ be the maximum of the orders of differential operators $T_{1}, \ldots, T_{k}$.

For each small $t>0$ we apply Lemma 2.2 to

$$
\Omega=D_{t}, \quad \varphi=\phi_{t}:=2(n+L) \mathcal{G}_{D}^{(t)}(\cdot, w)+|z|^{2}-\log \eta
$$

where

$$
\eta=-\log \frac{|z-\zeta|}{R_{D}}, R_{D}:=\operatorname{diam}(D)
$$

Then we get

$$
\begin{aligned}
\eta \mathscr{L}_{\phi_{t}}-\mathscr{L}_{\eta} & \geq \eta \mathscr{L}_{|z|^{2}}-\eta \mathscr{L}_{\log \eta}-\mathscr{L}_{\eta}=\eta \mathscr{L}_{|z|^{2}}-2 \mathscr{L}_{\eta}+\frac{\partial \eta \otimes \overline{\partial \eta}}{\eta} \\
& \geq Q+\frac{1}{2} \frac{\partial \eta \otimes \overline{\partial \eta}}{\eta}
\end{aligned}
$$

where

$$
Q=\frac{1}{2} \eta\left(\mathscr{L}_{|z|^{2}}+\frac{1}{2} \frac{\partial \eta \otimes \overline{\partial \eta}}{\eta^{2}}\right)
$$

The data of our $\bar{\partial}$-equation are defined by

$$
v:=\bar{\partial}\left(\lambda_{\varepsilon}\left(\frac{|z-\zeta|}{2 R}\right) f\right)
$$

Next we estimate the quantity $J_{Q, \phi_{t}}(v)$. Using $\bar{\partial} \eta=-\frac{\bar{\partial}|z-\zeta|}{|z-\zeta|}$ we compute

$$
\begin{aligned}
Q^{-1}(v) e^{-\phi_{t}}= & e^{-2(n+L) \mathcal{G}_{D}^{(t)}(\cdot, w)-|z|^{2}}\left|\lambda_{\varepsilon}^{\prime}\left(\frac{|z-\zeta|}{2 R}\right)\right|^{2}\left(\frac{|z-\zeta|}{2 R}\right)^{2} \eta Q^{-1}(\bar{\partial} \eta)|f|^{2} \\
\leq & e^{-2(n+L) \mathcal{G}_{D}(\cdot, w)} \frac{M^{2}}{\log ^{2} \log (1 / \varepsilon)}\left(\frac{\eta}{\log \frac{|z-\zeta|}{2 R}}\right)^{2}|f|^{2}, \\
& \text { using Lemma } 2.1 \text { and } \quad\left(\frac{|z-\zeta|}{2 R}\right)^{2} \eta Q^{-1}(\bar{\partial} \eta) \leq \eta^{2}, \\
& \text { as well as } \quad \mathcal{G}_{D}^{(t)}(\cdot, w) \geq \mathcal{G}_{D}(\cdot, w) .
\end{aligned}
$$


But $-\eta=\log \frac{|z-\zeta|}{R_{D}}=\log \frac{|z-\zeta|}{2 R}+\log \frac{2 R}{R_{D}}$, hence

$$
\left(\frac{\eta}{\log (|z-\zeta| / 2 R)}\right)^{2} \leq 2\left(1+\frac{\log ^{2}\left(2 R / R_{D}\right)}{\log ^{2}(|z-\zeta| / 2 R)}\right) \leq 4\left(1+\log ^{2}\left(2 R / R_{D}\right)\right)
$$

because, on $\operatorname{supp}(v)$ we have $|\log (|z-\zeta| / 2 R)| \geq \log 3$.

Altogether, this yields

$$
J_{Q, \phi_{t}}(v) \leq \frac{M_{1}}{\log ^{2} \log (1 / \varepsilon)} S(w, \varepsilon)\|f\|_{D \cap B(\zeta, R)}^{2}
$$

where $M_{1}>0$ is an unimportant constant and

$$
S(w, \varepsilon):=\exp \left(2(n+L) \max _{2 \varepsilon e^{-2 \varepsilon} R \leq|z-\zeta| \leq 2 R / 3}\left|\mathcal{G}_{D}(z, w)\right|\right) .
$$

By Lemma 2.2 we obtain a smooth solution $u_{t, w}$ to the equation $\bar{\partial}\left(\sqrt{\eta} u_{t, w}\right)$ $=v$ on $D_{t}$ such that

$$
\left\|u_{t, w}\right\|_{\phi_{t}}^{2} \leq 10 J_{Q, \phi_{t}}(v)
$$

Since $\eta e^{\phi_{t}} \leq e^{R_{D}^{2}}$ and hence $\left\|\sqrt{\eta} u_{t, w}\right\|^{2} \leq 10 e^{R_{D}^{2}} J_{Q, \phi_{t}}(v)$ we see that

$$
\widetilde{f}_{t, w}:=\lambda_{\varepsilon}\left(\frac{|z-\zeta|}{2 R}\right) f-\sqrt{\eta} u_{t, w} \in H^{2}\left(D_{t}\right)
$$

and

$$
\left\|\tilde{f}_{t, w}\right\|_{D_{t}} \leq\left(1+\frac{\sqrt{M_{2}}}{\log \log (1 / \varepsilon)} \sqrt{S(w, \varepsilon)}\right)\|f\|_{D \cap B(\zeta, R)}
$$

with $M_{2}:=10 e^{R_{D}^{2}} M_{1}$.

In a similar way as in [He1] and [DiHe2] we obtain a subsequence $\left(t_{j}\right)_{j}$ that tends to 0 and a smooth function $u_{w, \varepsilon}$ on $D$, such that in the weak- $\star-$ topology,

i) $\sqrt{\eta} u_{t_{j}, w} \longrightarrow \sqrt{\eta} u_{w, \varepsilon}$, as $j \longrightarrow \infty$

and

ii) $\widetilde{f}_{t_{j}, w} \longrightarrow \widetilde{f}_{w}:=\lambda_{\varepsilon}\left(\frac{|z-w|}{R}\right) f-\sqrt{\eta} u_{w, \varepsilon}$

Furthermore, using (i), we have

$$
\begin{gathered}
\left\|\sqrt{\eta} u_{w, \varepsilon}\right\|_{D}^{2} \leq 10 e^{R_{D}^{2}} \int_{D} \eta(z)\left|u_{w, \varepsilon}(z)\right|^{2} e^{-2(n+L) \mathcal{G}_{D}(z, w)-|z|^{2}} d^{2 n} z \\
\leq \frac{M_{2}}{\log \log (1 / \varepsilon)} S(w, \varepsilon)\|f\|_{D \cap B(\zeta, R)}^{2}
\end{gathered}
$$


As a consequence, all derivatives of $u_{w, \varepsilon}$ up to order $L$ vanish at the point $w$, and $\widetilde{f}_{w}$ is holomorphic on $D$ and belongs to $H^{2}(D)$, its $L^{2}$-norm is

$$
\left\|\tilde{f}_{w}\right\|_{D} \leq\left(1+\frac{\sqrt{M_{2}}}{\log \log (1 / \varepsilon)} \sqrt{S(w, \varepsilon)}\right)\|f\|_{D \cap B(\zeta, R)} .
$$

Finally we bring the hypothesis (1.1) into the picture. Let $\varepsilon>0$ be arbitrary. Then we can choose $\delta=\delta(\varepsilon, R)>0$ so small, that

$$
A_{w} \subset D \cap B\left(\zeta, 2 \varepsilon e^{-2 \varepsilon} R\right)
$$

whenever $w \in D \cap B(\zeta, \delta)$. Then $A_{w}$ is disjoint with the support of $v$, and hence $S(w, \varepsilon) \leq e^{2(n+L)}$ for such points $w$.

Given a arbitrarily small number $s>0$, we choose $\varepsilon>0$ so small, that $\left(\sqrt{M_{2}} e^{(n+L)}\right) /(\log \log (1 / \varepsilon))<s$, and then $\delta=\delta(\varepsilon, R)$ as described before.

The main lemma is proved.

\section{$\S 3$. Application to plurisubharmonic peak points}

We deal with the question, under which conditions we can prove

$$
\lim _{w \rightarrow \zeta} \operatorname{diam}\left(A_{w}\right)=0
$$

Our result is:

LEMmA 3.1. Suppose that D has a $\mathscr{C}^{2}$-smooth boundary, and $\zeta \in \partial D$ is a plurisubharmonic peak point and there exists a plurisubharmonic peaking function $\psi \in \mathscr{C}^{0}(\bar{D})$ that is even Hölder continuous at $\zeta$, more precisely: We have, with some constants $C, \lambda>0$, that $|\psi| \leq C|\cdot-\zeta|^{\lambda}$. Then

$$
\lim _{\substack{w \rightarrow \zeta \\ \Lambda}} \operatorname{diam}\left(A_{w}\right)=0
$$

(meaning that in the limit on the left side, $w$ is supposed to tend to $\zeta$ nontangentially).

This will follow from

Lemma 3.2. Suppose that $D$ has a $\mathscr{C}^{2}$-smooth boundary. Then, for a sufficiently small positive number $\alpha$, the following holds: If $r_{0}>0$ is a fixed 
number, then there exists a constant $C^{*}>0$, such that for any $x, y \in D$ with $|x-y|>r_{0}$ one has

$$
\begin{aligned}
& \left|\mathcal{G}_{D}(x, y)\right| \leq \\
& \begin{cases}C^{*} \delta_{D}(y)^{3 \alpha / 8} & \text { for } \delta_{D}(x) \leq \delta_{D}(y)^{3 / 2} \\
C^{*} \delta_{D}(y)^{\alpha / 20 n} & \text { for } \delta_{D}(x) \geq \delta_{D}(y)^{2 / 3} \\
C^{*}\left(\delta_{D}(y)^{-3 \alpha / 8 n}\left|\mathcal{G}_{D}(y, x)\right|^{1 / n}+\delta_{D}(y)^{\alpha / 8 n}\right) & \text { for } \delta_{D}(y)^{3 / 2} \leq \delta_{D}(x) \leq \delta_{D}(y)^{2 / 3}\end{cases}
\end{aligned}
$$

One should note here, that the point $x$ is not supposed to stay within any compact subset of $D$, hence the lemma is not simply a corollary of Thm. 1.4 from [DiHe2].

Proof of the lemma. If $\alpha>0$ is small, we can choose a Hölder- $\alpha$ continuous plurisubharmonic exhaustion function $\varrho: D \longrightarrow(-1,0)$, such that

$$
\widetilde{C}_{2} \delta_{D}^{\alpha} \leq-\varrho \leq \widetilde{C}_{1} \delta_{D}^{\alpha}
$$

with $\widetilde{C}_{1}, \widetilde{C}_{2}>0$, and $\alpha \in(0,1)$. This follows from [DiFo1].

We make use of two estimates on the Green function, that were obtained in [DiHe2]:

(i) There exists a constant $C_{1}>0$, such that for any compact set $K \subset \subset D$

$$
\sup _{w \in K}\left|\mathcal{G}_{D}(z, w)\right| \leq C_{1}\left(\frac{\delta_{D}(z)}{\delta_{D}(K)}\right)^{\alpha}\left(\log \frac{R_{D}}{\delta_{D}(w)}\right), \text { if } \delta_{D}(z)<\frac{1}{2} \delta_{D}(K)
$$

and

(ii)

$$
\begin{gathered}
\sup _{z \in K}\left|\mathcal{G}_{D}(z, w)\right| \leq \\
C_{1}\left(\left(\frac{\delta_{D}(w)^{3 / 4}}{\delta_{D}(K)}\right)^{\alpha / n}\left(\log \frac{R_{D}}{\delta_{D}(w)}\right)^{1 / n}+\delta_{D}(w)^{\alpha / 8 n}(w)\left|\log \delta_{D}(w)\right|\right),
\end{gathered}
$$

whenever $w \in D \backslash K, \delta_{D}(w)<\frac{1}{C_{1}} \delta_{D}(K)$

Let $x, y \in D$ be arbitrary points. Let $\delta_{0} \ll 1$. We may suppose that $\delta_{D}(y) \leq \delta_{0}$, otherwise the left-hand side of the claimed estimate is $\leq \log \frac{R_{D}}{\delta_{0}}$, 
while the right side is bounded away from zero, and there is nothing to prove.

(A) Assume, that $\delta_{D}(x) \leq \delta_{D}(y)^{3 / 2}$.

We apply (3.2) to $z=x$ and $K=\{y\}$. This yields

$$
\begin{aligned}
\left|\mathcal{G}_{D}(x, y)\right| & \leq C_{1}\left(\frac{\delta_{D}(x)}{\delta_{D}(y)}\right)^{\alpha}\left(\log \frac{R_{D}}{\delta_{D}(y)}\right) \\
& \leq \delta_{D}(y)^{\alpha / 2}\left(\log \frac{R_{D}}{\delta_{D}(y)}\right) \leq C_{2} \delta_{D}(y)^{3 \alpha / 8}
\end{aligned}
$$

We turn to the next case:

(B) Assume that $\delta_{D}(x) \geq \delta_{D}(y)^{2 / 3}$.

We apply (3.4) with $K=\{x\}, w=y$. This gives us

$$
\begin{aligned}
& \left|\mathcal{G}_{D}(x, y)\right| \\
& \quad \leq C_{1}\left(\left(\frac{\delta_{D}(y)^{3 / 4}}{\delta_{D}(x)}\right)^{\alpha / n}\left(\log \frac{R_{D}}{\delta_{D}(y)}\right)^{1 / n}+\delta_{D}(y)^{\alpha / 8 n}(y)\left|\log \delta_{D}(y)\right|\right) \\
& \quad \leq C_{1}\left(\delta_{D}(y)^{\alpha / 12 n}\left(\log \frac{R_{D}}{\delta_{D}(y)}\right)^{1 / n}+\delta_{D}(y)^{\alpha / 8 n}(y)\left|\log \delta_{D}(y)\right|\right) \\
& \quad \leq C_{2} \delta_{D}(y)^{\alpha / 20 n} .
\end{aligned}
$$

Now we come to

(C) The case $\delta_{D}(y)^{3 / 2} \leq \delta_{D}(x) \leq \delta_{D}(y)^{2 / 3}$.

This case can be treated by the methods applied in the proof of Theorem 1.4 of [DiHe2]. For the reader's convenience we recall the main steps in the proof.

First we show, that, with some (unimportant) constant $C_{3}>0$

$$
\begin{aligned}
& \mathcal{G}_{D}(s, y) \leq \frac{1}{2} \mathcal{G}_{D}(x, y)+C_{3} \delta_{D}(y)^{\alpha / 8 n} \\
& \quad \quad \text { if } \delta_{D}(x)<C_{3}^{-1}, s \in B\left(x, R_{D} \exp \left(-\delta_{D}(x)^{-\alpha / 4 n}\right)\right) .
\end{aligned}
$$

For this we put

$$
\sigma=-(-\varrho)^{1 / 8 n}
$$

Then we have, with some constant $C_{4}>0$ :

$$
\left|\sigma\left(z^{\prime}\right)-\sigma\left(z^{\prime \prime}\right)\right| \leq C_{4}\left|z^{\prime}-z^{\prime \prime}\right|^{\alpha / 8 n}, \text { for } z^{\prime}, z^{\prime \prime} \in D
$$


With a suitable small $\varepsilon^{0}>0$ we get for all $0<\varepsilon_{1}<\varepsilon^{0}$ :

(i)

$$
\log \varepsilon_{1}^{1 / \alpha}-\log R_{D}-\frac{\log C_{4}}{\alpha}-\varepsilon_{1}>-\frac{1}{\varepsilon_{1}}
$$

(ii)

$$
\log \frac{\varepsilon_{1}^{1 / \alpha}}{R_{D}}>\left(1-\varepsilon_{1}\right) \log \varepsilon_{1}^{1+\frac{1}{\alpha}}
$$

Furthermore, we put

$$
\varepsilon:=|\sigma(y)|
$$

and

$$
t=t(y):=\left(\frac{1}{2 C_{4}^{8 n / \alpha}} \delta_{D}(y)\right)^{1 / \varepsilon} \varepsilon^{8 n(1-\varepsilon) / \alpha \varepsilon} .
$$

Then we have for small enough $\delta_{0}$ :

$$
t<\min \left\{\left(\frac{\varepsilon^{3}}{C_{4}}\right)^{8 n / \alpha}, \frac{1}{2} \delta_{D}(y)^{3 / 2}\right\}
$$

We want to regularize the Green function. For this we choose a radially symmetric function $\alpha_{1} \geq 0$ in the class $\mathscr{C}_{0}^{\infty}$ which is supported in the unit ball, with $L^{1}$-norm equal to 1 , and put

$$
\psi_{y}^{t}:=\mathcal{G}_{D}(\cdot, y) \star \alpha_{t},
$$

where $\alpha_{t}(v)=t^{-2 n} \alpha_{1}(v / t)$, and $t$ is as defined above.

Let

$$
\phi_{y}(s):=(1-\varepsilon) \log \left(\varepsilon^{8 n / \alpha}|s-y|\right)-\varepsilon-8 n \frac{\log C_{4}}{\alpha} .
$$

Then the following function $v_{y}$ becomes plurisubharmonic on $D$

$$
v_{y}(s):=\left\{\begin{array}{lll}
\varepsilon^{-2} \sigma(s) & \text { for } & \sigma(s)>-\varepsilon^{3} \\
\max \left\{\psi_{y}^{t}(s)-\varepsilon, \varepsilon^{-2} \sigma(s)\right\} & \text { for } & -\varepsilon \leq \sigma(s) \leq-\varepsilon^{3} \\
\max \left\{\psi_{y}^{t}(s)-\varepsilon, \phi_{y}(s)\right\} & \text { for } & \sigma(s)<-\varepsilon,|s-y| \geq t \\
\phi_{y}(s) & \text { for } & \sigma(s)<-\varepsilon,|s-y|<t
\end{array} .\right.
$$

Obviously $v_{y} \leq(1-\varepsilon) \mathcal{G}_{D}(\cdot, y)$.

Using the hypothesis of case $(\mathrm{C})$, we get

$$
\begin{aligned}
|s-y|>t, \text { and } \delta_{D}(s) & \geq \frac{1}{2} \delta_{D}(y)^{3 / 2}>t \\
\text { for any } s & \in B\left(x, R_{D} \exp \left(-\delta_{D}(x)^{-\alpha / 4 n}\right)\right)
\end{aligned}
$$


(recall that $\left.|x-y| \geq r_{0} !\right)$. Moreover

$$
\psi_{y}^{t}(x) \leq \varepsilon+v_{y}(x) \leq(1-\varepsilon) \mathcal{G}_{D}(x, y)+\varepsilon .
$$

From this we obtain:

$$
\begin{aligned}
\mathcal{G}_{D}(s, y) & \leq \psi_{y}^{t}(s) \leq \psi_{y}^{t}(x)+\psi_{y}^{t}(s)-\psi_{y}^{t}(x) \\
& \leq(1-\varepsilon) \mathcal{G}_{D}(x, y)+\varepsilon+\psi_{y}^{t}(s)-\psi_{y}^{t}(x), \text { using }
\end{aligned}
$$

Again, we can prove that

$$
\begin{aligned}
\left|\psi_{y}^{t}(s)-\psi_{y}^{t}(x)\right| & \leq C_{5}\left(\log (1 / \varepsilon) \frac{|s-x|}{t}\right. \\
& \leq C_{5} R_{D}\left(\log (1 / \varepsilon) \frac{\exp \left(-\delta_{D}(x)^{-\alpha / 4 n}\right)}{t} \leq C_{6} \varepsilon\right.
\end{aligned}
$$

The latter inequality is obtained by means of $\delta_{D}(x) \leq \delta_{D}(y)^{2 / 3}$ together with (3.1): We have

$$
\begin{aligned}
\exp \left(-\delta_{D}(x)^{-\alpha / 4 n}\right) & \leq \exp \left(-\delta_{D}(y)^{-\alpha / 6 n}\right) \\
& \leq \exp \left(-\left(\widetilde{C}_{2} /|\varrho(y)|\right)^{1 / 6 n}\right)=\exp \left(-\frac{\widetilde{C}_{2}^{1 / 6 n}}{\varepsilon^{4 / 3}}\right)
\end{aligned}
$$

On the other hand,

$$
\begin{aligned}
t & =\left(2 C_{4}^{8 n / \alpha}\right)^{-1 / \varepsilon} \delta_{D}(y)^{1 / \varepsilon} \varepsilon^{\frac{8 n(1-\varepsilon)}{\alpha \varepsilon}} \geq\left(2 C_{4}^{8 n / \alpha}\right)^{-1 / \varepsilon}\left(\frac{|\varrho(y)|}{\widetilde{C}_{1}}\right)^{1 / \alpha \varepsilon} \varepsilon^{\frac{8 n(1-\varepsilon)}{\alpha \varepsilon}} \\
& =\left(2^{\alpha} \widetilde{C}_{1} C_{4}^{8 n}\right)^{-1 / \alpha \varepsilon} \varepsilon^{\frac{8 n(2-\varepsilon)}{\alpha \varepsilon}}=\exp \left(-\frac{C_{6}+C_{7} \log (1 / \varepsilon)}{\varepsilon}\right)
\end{aligned}
$$

with unimportant constants $C_{6}, C_{7}>0$. This proves (3.4).

Let $\eta>0$ and

$$
U_{x, \eta}:=\max \left\{\mathcal{G}_{D}(\cdot, x),-\eta\right\}
$$

Then we have (using an inequality of Blocki ([Blo $]$ ))

$$
\int_{D}\left|\mathcal{G}_{D}(\cdot, y)\right|\left(d d^{c} U_{x, \eta}\right)^{n} \leq(2 \pi)^{n} n !^{1 / n} \eta^{n-1 / n}\left|\mathcal{G}_{D}(y, x)\right|^{1 / n}
$$


We note that the measure $\left(d d^{c} U_{x, \eta}\right)^{n}$ is supported in the set $\left\{\mathcal{G}_{D}(\cdot, x)=\right.$ $-\eta\} \subset B\left(x, R_{D} e^{-\eta}\right)$ and its total mass is equal to $(2 \pi)^{n}$. If we choose $\eta=\delta_{D}(x)^{-\alpha / 4 n}$, we can apply (3.4). This gives us

$$
\int_{D}\left|\mathcal{G}_{D}(\cdot, y)\right|\left(d d^{c} U_{x, \eta}\right)^{n} \geq(2 \pi)^{n}\left(\frac{1}{2}\left|\mathcal{G}_{D}(x, y)\right|-C_{3} \delta_{D}(y)^{1 / 8 n}\right)
$$

and therefore (by the choice of $\varepsilon$ ):

$$
\left|\mathcal{G}_{D}(x, y)\right| \leq C_{7}\left(\delta_{D}(x)^{-\alpha / 4 n}\left|\mathcal{G}_{D}(y, x)\right|^{1 / n}+\delta_{D}(y)^{\alpha / 8 n}\right)
$$

If we, finally, observe that $\delta_{D}(x)^{-\alpha / 4 n} \leq \delta_{D}(y)^{-3 \alpha / 8 n}$, we obtain the desired estimate.

The next lemma is implicit in [DiHe2]:

Lemma 3.3. Assume that $\zeta \in \partial D$ admits a plurisubharmonic peak function $\psi \in \mathscr{C}^{0}(\bar{D})$. If $\rho_{1}>0$ and $z, w \in D$ are points such that $|w-\zeta|<\rho_{1} / 3$ and $|z-w| \geq \rho_{1}$, then, with

$$
C_{1}\left(\rho_{1}, \psi\right):=2 \frac{\log \left(3 R_{D} / \rho_{1}\right)}{\min _{x \in D:|x-\zeta| \geq \rho_{1} / 3}|\psi(x)|}
$$

we can estimate the Green function by

$$
\mathcal{G}_{D}(w, z) \geq C_{1}\left(\rho_{1}, \psi\right) \psi(w)
$$

With this lemma we can easily give the

Proof of Lemma 3.1. Assume that the plurisubharmonic peak function $\psi$ at $\zeta$ satisfies

$$
|\psi(z)| \leq C^{\prime}|z-\zeta|^{\lambda}
$$

with some $\lambda \in(0,1]$. If the claim were false, we could find a sequence $\left(w_{j}\right)_{j}$ of points such that $\delta_{D}\left(w_{j}\right) \geq c \cdot\left|w_{j}-\zeta\right|$ (with a constant $c>0$ ) and $\lim _{j \rightarrow \infty} w_{j}=\zeta$. Furthermore we find points $z_{j} \in A_{w_{j}}$, such that $\left|z_{j}-w_{j}\right| \geq r_{0}$ with some $r_{0}>0$. Now lemma 3.3 applies, and we get

$$
\left|\mathcal{G}_{D}\left(w_{j}, z_{j}\right)\right| \leq C^{\prime \prime}\left|\psi\left(w_{j}\right)\right| \leq C^{\prime \prime \prime} \delta_{D}\left(w_{j}\right)^{\lambda}
$$


In Lemma 3.1 we can choose $\alpha$ sufficiently small. Then, by means of (3.6), with $x=z_{j}, y=w_{j}$, Lemma 3.2 implies

$$
\left|\mathcal{G}_{D}\left(z_{j}, w_{j}\right)\right| \longrightarrow 0, \text { as } j \longrightarrow \infty \text {. }
$$

But this contradicts the assumption $z_{j} \in A_{w_{j}}$ which says, that the left side is $\geq 1$ for all $j$. Lemma 3.1 is therefore proved.

The proof of Theorem 1.1 and the Main Lemma imply the following application

TheOREM 3.1. Let $D \subset \subset \mathbb{C}^{n}$ be pseudoconvex with a $\mathscr{C}^{2}$-smooth boundary, and $\zeta \in \partial D$ a point which admits a plurisubharmonic peak function that is even Hölder continuous at $\zeta$.

If $M$ and $Y$ have the meanings as explained before Theorem 1.1, then for any radius $R>0$ we have for non-tangential approach of $w$ towards $\zeta$ :

$$
\lim _{\Lambda \ni w \rightarrow \zeta} \frac{\left\|\mathcal{M}_{D}(\cdot ; M, Y ; w)\right\|}{\left\|\mathcal{M}_{D \cap B(\zeta, R)}(\cdot ; M, Y ; w)\right\|}=1
$$

\section{REFERENCES}

[BeFo] E. Bedford and J.E. Fornæss, Biholomorphic maps of weakly pseudoconvex domains, Duke Math. J., 45 (1978), 711-719.

[Blo] Z. Błocki, Estimates for the complex Monge-Ampère operator, Bull. Pol. Acad. Sci., 41 (1993), 151-157.

[BlPf] Z. Błocki and P. Pflug, Hyperconvexity and Bergman completeness, Nagoya Math. J., 151 (1998), 221-225.

[CCW] M. Carlehed, U. Cegrell and F. Wikström, Jensen measures, Hyperconvexity and Boundary Behavior of the Pluricomplex Green's Function, Ann. Pol. Math., $\mathbf{7 1}$ (1999), 87-103.

[Di] K. Diederich, Randverhalten der Bergmanschen Kernfunktion und Metrik in streng pseudokonvexen Gebieten, Math. Ann., 198 (1970), 1-36.

[DiFo1] K. Diederich and J.E. Fornæss, Pseudoconvex domains: Bounded strictly plurisubharmonic exhaustion functions, Inv. Math., 39 (1977), 129-141.

[DiFo2] - Pseudoconvex domains: Existence of Stein neighborhoods, Duke Math. J., 44 (1977), 641-662.

[DiFoHe] K. Diederich, J.E. Fornæss and G. Herbort, Boundary behavior of the Bergman metric, Proc. Pure Math., 41 (1984), 59-67.

[DiHe1] K. Diederich and G. Herbort, Pseudoconvex domains of semiregular type, Contributions to Complex Analysis and Analytic Geometry (Skoda,H.-Trépreau, Eds. ), Aspects of Mathematics, E26 (1994), 127-162. 
[DiHe2] — Quantitative estimates for the Green function and an application to the Bergman metric, Ann. Inst. Fourier (Grenoble), 50 (2000), 1205-1228.

[DiHe3] An alternative proof of a theorem of T. Ohsawa, Michigan Mathematics J., 46 (1999), 347-360.

[DiOh1] K. Diederich and T. Ohsawa, Moment problems for weighted Bergman kernels, Complex analysis and geometry (Paris, 1997), Progress in Mathematics, 188 (2000), 111-122.

[DiOh2] _ An estimate for the Bergman distance on pseudoconvex domains, Ann. of Math., 141 (1995), 181-190.

[De] J.-P. Demailly, Mesures de Monge-Ampère et mesures pluriharmoniques, Math. Z., 194 (1987), 519-564.

[FoSi] J.E. Fornæss and N. Sibony, Construction of p.s.h. functions on weakly pseudoconvex domains, Duke Math. J., 58 (1989), 633-656.

[He1] G. Herbort, The Bergman metric on hyperconvex domains, Math. Z., 232 (1999), 183-196.

[He2] Boundary behavior of the pluricomplex Green function on pseudoconvex domains with a smooth boundary, Internatl. J. Math., 11 (2000), 509-522.

[Hoer] L. Hörmander, $L^{2}$-estimates and existence theorems for the $\bar{\partial}$-operator, Acta Math., 113 (1965), 89-152.

[Kli] M. Klimek, Extremal plurisubharmonic functions and invariant pseudodistances, Bull. Soc. Math. France, 113 (1985), 231-240.

[KrYu] S. Krantz and J. Yu, On the Bergman invariant and curvatures of the Bergman metric, Illinois J. Math., 40 (1996), 226-244.

$[\mathrm{McN}]$ J. McNeal, Lower bounds on the Bergman metric near a point of finite type, Ann. Math., 136 (1992), 339-360.

[Nik] N. Nikolov, Localization of invariant metrics, Arch. Math., 79 (2002), 67-73.

[Oh1] T. Ohsawa, Boundary behavior of the Bergman kernel function on pseudoconvex domains, Publ. R.I.M.S. Kyoto University, 20 (1984), 897-902.

[OhTa] T. Ohsawa and K. Takegoshi, On the extension of $L^{2}$-holomorphic functions, Math. Z., 195 (1987), 197-204.

[Si] N. Sibony, Une classe de domaines pseudoconvexes, Duke Math. J., 55 (1987), 299-319.

$[\mathrm{Yu}] \quad$ J. Yu, Peak functions on weakly pseudoconvex domains, Indiana Univ. Math. J., 43 (1994), 1271-1295.

Fachbereich C - Mathematik und Naturwissenschaften

Bergische Universität Wuppertal

Gaußstraße 20

D-42097 Wuppertal

Germany

gregor@math.uni-wuppertal.de 